\title{
In vitro Antibacterial Activity of Chitosan Nanoparticles against Mycobacterium tuberculosis
}

\author{
Giftania Wardani' ${ }^{1}$ Mahmiah$^{1}$, Sri Agus Sudjarwo²
}

\section{Giftania Wardani', Mahmiah', Sri Agus Sudjarwo 2}

'Department of Pharmacy Biology, Faculty of Pharmacy, Hang Tuah University, Surabaya, INDONESIA. ${ }^{2}$ Department of Pharmacology, Faculty of Veterinary Medicine, Airlangga University, Surabaya-60115, INDONESIA.

\section{Correspondence}

Sri Agus Sudjarwo

Department of Pharmacology, Faculty of Veterinary Medicine, Airlangga University, Surabaya, INDONESIA.

Phone no: 62-315992785

E-mail: ags158@yahoo.com

History

- Submission Date: 10-10-2017;

- Review completed: 08-11-2017;

- Accepted Date: 11-11-2017

DOI : 10.5530/pj.2018.1.27

Article Available online

http://www.phcogj.com/v10/i1

Copyright

(C) 2018 Phcog.Net. This is an openaccess article distributed under the terms of the Creative Commons Attribution 4.0 International license.

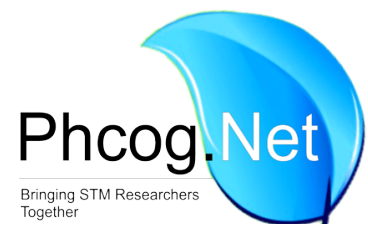

\begin{abstract}
Background: Chitosan nanoparticles have gained growing interest for nanomedicine, biomedical engineering and development of new therapeutic with improved bioavailability, increased sensitivity and specificity, and reduced toxicity. Objective: The aim of the present study is to synthesis of the chitosan nanoparticles for antimycobacterial applications. Methods: Chitosan were isolated from the shrimp shell. Tripolyphosphate (TPP) will be used to prepare chitosan nanoparticles by ionotropic gelation method. The size and morphology of the chitosan nanoparticle was analyzed by scanning electron microscope (SEM). The broth microdilution method is used to determine the minimal inhibitory concentration (MIC) and minimal bactericidal concentration (MBC) of nanoparticle chitosan on strain M. tuberculosis H37Rv. Results: The SEM micrographs of the nano-magnetic chitosan showed that they were approximately uniform spheres and the rough surface morphology, have a solid dense cubical or rectangular structure. Using the broth microdilution susceptibility method, chitosan nanoparticle was found to have the antimycobacterial effects with a MIC value of $1200 \mu \mathrm{g} / \mathrm{mL}$ whilst MBCs value of $2400 \mu \mathrm{g} / \mathrm{mL}$ for $M$. tuberculosis H37Rv. Conclusion: The conclusion from the study was chitosan nanoparticle have potential as a source of lead compounds that may be developed further into antimycobacterial drugs.

Key words: Chitosan nanoparticle, M. tuberculosis H37Rv, Minimal inhibitory Concentration, Minimal bactericidal concentration.
\end{abstract}

\section{INTRODUCTION}

Tuberculosis (TB) is the disease caused by Mycobacterium tuberculosis which $99 \%$ of cases were reported in developing countries and over 8 million new cases of infection diagnosed each year and is responsible for more than two million deaths per year. The global incidence rate for TB is growing each year by approximately $1.1 \%$ and the number of cases by about $2.4 \%$. Resistance to anti-TB drugs continued to be recognized as a clinical problem through the latter part of the 21st century. As a result, MDR and XDR TB are now becoming a major threat to health worldwide, accounting for almost $3 \%$ of all newly reported cases of TB. ${ }^{1}$ Due to increased drug resistant strains of bacteria such as $M$. tuberculosis and methicillin resistant Staphylococcus aureus there has been renewed interest in natural products as potential sources of novel antibiotics. $^{2,3}$

The use of natural products as medicines is well known in rural areas of many developing countries. The natural products claim that their medicine is cheaper, more effective and impart least side effects as compared to synthetic medicines. ${ }^{4,5}$ In this regard, increasing investigation has been given to chitosan and its derivatives. Chitosan is derived from naturally occurring sources, including the exoskeletons of insects, arthropods such as crustacean shells, shellfish like shrimp, prawns, crabs, and beaks of cephalopods as well as cell walls of fungi. ${ }^{6,7}$ Chitosan and its derivatives are undisputed biomolecules of great potential by their polyelectrolite properties, including the presence of reactive functional groups, gel-forming ability, high adsorption capacity, complete biodegradability, Antimicrobial, and antifungi, even anti-tumor influence. ${ }^{8,9}$ Chitosan is widely recognized for its potent antimicrobial activity with, broad spectrum, and high killing rate but low toxicity toward mammalian cells. Although the mode of antimicrobial action of chitosan is not completely understood, it is well established that the molecular structure of chitosan is prerequisite for its antimicrobial activity. ${ }^{10,11}$ Chitosan has been investigated as an antimicrobial material against a wide range of target organisms like algae, bacteria, yeasts, and fungi in experiments involving in vivo and in vitro. ${ }^{12}$

Some researcher has stated that chitosan generally showed stronger effects for g-positive bacteria (e.g. Listeria monocytogenes, Bacillus megaterium, B. cereus, Staphylococcus aureus, Lactobacillus plantarum, L. brevis, L. bulgaris, etc.) than for g-negative bacteria (e.g. E. coli, Pseudomonas fluorescens, Salmonella typhymurium, Vibrio parahaemolyticus, etc.). ${ }^{10,11,12}$ The antibacterial activity of chi-

Cite this article: Wardani G, Mahmiah, Sudjarwo SA. In vitro Antibacterial Activity of Chitosan Nanoparticles against Mycobacterium tuberculosis. Pharmacog J. 2018;10(1):162-66. 
tosan is influenced by a number of factors that include the type of chitosan, the degree of chitosan polymerization and some of its other physicochemical properties.

Over the last decade, the modifications of chitosan have been reported. The modification of chitosan nanoparticle has received much attention in relation to their potential application, especially from a pharmaceutical viewpoint. ${ }^{13,14}$ Nanoparticle chitosan has gained growing interest due to its biocompatibility, biodegradability, high permeability, cost-effectiveness, non-toxic property and excellent film forming ability. Moreover, its ability to enhance the penetration of large molecules across a mucosal surface and its recognition as muco adhesivity chitosan. ${ }^{15,16}$ However, the antibacterial effect of chitosan nanoparticles has seldom been reported elsewhere. The unique character of chitosan nanoparticles for their small size and quantum size effect could make chitosan nanoparticles exhibit superior activities. The aim of the present study is to a synthesis of the chitosan nanoparticles for antimycobacterial applications. Chitosan nanoparticles have potential role as polymeric platforms for the development of new pharmacological and therapeutic drug release systems with improved biodistribution and increased specificity and sensitivity and reduced pharmacological toxicity

\section{MATERIALS AND METHODS}

\section{Preparation of chitosan}

The typical production of chitosan from crustacean shell generally consists of three basic steps: demineralization, deproteinization, and deacetylation. ${ }^{6}$ The shells were demineralized by agitating continuously with $5 \% \mathrm{HCl}$ at the ratio of $1: 15(\mathrm{w} / \mathrm{v}$, shell to solution) $36 \mathrm{~h}$ at room temperature. The demineralized shells were treated with $5 \% \mathrm{NaOH}$ solution at the ratio of shell to solution of $1: 10(\mathrm{w} / \mathrm{v})$ at $90-95^{\circ} \mathrm{C}$ for $6 \mathrm{~h}$. The deproteinized shells were filtered and washed with tap water until $\mathrm{NaOH}$ was removed completely, then dried overnight in an oven at $55-60^{\circ} \mathrm{C}$. The shells were filtered and washed with tap water until became neutral. Then deacetylation of chitosan was carried out by hydrolyzing with $80 \% \mathrm{NaOH}$ at the ratio of $1: 20$ (w/v, chitin to solvent) at $90-95^{\circ} \mathrm{C}$ for $5 \mathrm{~h}$. This product was washed with tap water until it became neutral and dried overnight at $55-60^{\circ} \mathrm{C}$. In the preparation of chitosan solutions, $1.0 \%(\mathrm{w} / \mathrm{v})$ chitosans were dispersed in a $1.0 \%(\mathrm{v} / \mathrm{v})$ acetic acid solution.

\section{Preparation of chitosan nanoparticles}

In the present work, the chitosan nanoparticles were synthesized from the chitosan using sodium tripolyphosphate as a crosslinking agent by ionotropic gelation method..$^{17}$ Initially in order to create the homogeneous chitosan solution, about $1.5 \mathrm{~g}$ of chitosan dissolved in $200 \mathrm{ml}$ of $2 \%$ acetic acid solution was kept under magnetic stirring process for about $20 \mathrm{~min}$. Then to the above prepared chitosan solution, $0.8 \mathrm{~g}$ of sodium tripolyphosphate dissolved in $107 \mathrm{ml}$ of conductivity water was added drop wise and stirred well for about $30 \mathrm{~min}$ to reach equilibrium. A milky colored emulsion like appearance of chitosan nanoparticles was formed upon the ionic cross linking between the sodium tripolyphosphate and chitosan solution. After reaching equilibrium, The suspension was formed in above mentioned conditions. The nanoparticles were separated by centrifugation at $20,000 \mathrm{~g}$ and $14^{\circ} \mathrm{C}$ for $30 \mathrm{~min}$, freezedried and stored at $5 \pm 3^{\circ} \mathrm{C}$.

\section{Electron microscopy analysis}

After the preparation of the synthesized chitosan nanoparticles, the characterization of the nanoparticle was examined by scanning electron microscope (SEM). ${ }^{18}$ The particle size and morphology of synthesized nano materials were determined using a field emission scanning electron microscope (FE-SEM, 15 kV, model 54160, Hitachi, Japan).

\section{Culture and preparation of Mycobacterium tuberculosis}

Mycobacterium tuberculosis strains $\mathrm{H} 37 \mathrm{Rv}$ were obtained from the Institute of Tropical Disease, Airlangga University, Surabaya, Indonesia. Mycobacterium tuberculosis was cultured at $37^{\circ} \mathrm{C}$ in Middlebrook 7H9 broth (Becton Dickinson, Sparks, MD) supplemented with $0.2 \%$ glycerol (Sigma Chemical Co.,St. Louis, MO) and 10\% OADC (oleic acidalbumin-dextrose-catalase; Becton Dickinson) until logarithmic growth was reached. Each culture was mixed with a sufficient volume of sterile supplemented Middlebrook 7H9 broth to achieve a turbidity equivalent to that of McFarland's No. 1 standard. To obtain the test inoculum, this suspension was further diluted 1:50 with the same culture medium to approximately $6 \times 10^{6}$ colony-forming units $(\mathrm{CFU}) / \mathrm{mL}$ immediately before use. A change in color from blue to pink indicated the growth of bacteria, and the MIC was defined as the lowest concentration of drug that prevented this change in color.

\section{Minimum Inhibitory Concentration determination by Resazurin Microtiter Plate Assay (REMA) method}

REMA was performed with minor modifications. ${ }^{19,20}$ The resazurin microtiter assay (REMA) plate method was performed in 7H9-S medium containing Middlebrook broth, $0.1 \%$ Casitone, and 0.5\% glycerol and supplemented with oleic acid, albumin, dextrose, and catalase (BectonDickinson). Briefly, $100 \mu \mathrm{L}$ of Middlebrook 7H9 broth was dispensed into each well of the microtitre plate. Serial four-fold dilutions of chitosan nanoparticles $(0 ; 150 ; 300 ; 600 ; 1200$; and $2400 \mu \mathrm{g} / \mathrm{mL})$ and standard antimycobacterial drugs were made in the plate. Mycobacterium tuberculosis strains $\mathrm{H} 37 \mathrm{Rv}$ suspension $(100 \mu \mathrm{L})$ containing approximately $6 \times 10^{6} \mathrm{CFU} / \mathrm{mL}$ was added to all the wells. Sterility control and growth control were also included. The plate was wrapped in aluminium foil and incubated at $37^{\circ} \mathrm{C}$ for seven days. After completion of the incubation period, $30 \mu \mathrm{L}$ resazurin solution $(100 \mu \mathrm{g} / \mathrm{mL})$ was added to each well and plate was again wrapped in aluminium foil and incubated overnight. The plate was then observed for change in color. The color change from blue to pink or colorless indicated growth of the bacteria. The lowest concentration of drug or extract that prevented color a change from blue to pink was taken as the upper limit for Minimum Inhibitory Concentration (MIC) range; the highest drug/extract concentration that showed change in color from blue to pink was considered the lower limit. All evaluations were carried out in quadruplicate.

\section{Minimum Bactericidal Concentration determination by using the paper disc method}

Screening of chitosan nanoparticles and its solvents for antimycobacterial activity against Mycobacterium tuberculosis strain $\mathrm{H} 37 \mathrm{Rv}$ were done using the paper disc method. ${ }^{21,22}$ For the treatment, each of $0 ; 150 ; 300$; $600 ; 1200 ; 2400 \mu \mathrm{g} / \mathrm{mL}$ chitosan nanoparticles solutions were slowly absorbed into the sterilized paper disc (diameter: $8 \mathrm{~mm}$, Watman, England) and adhered to the surface of the plate on which Mycobacterium tuberculosis strains $\mathrm{H} 37 \mathrm{Rv}$ at a concentration of $10^{6} \mathrm{CFU} / \mathrm{ml}$ had been inoculated in Middlebrook 7H9 broth. Sterilized distilled water was used as a control. After culturing for $24 \mathrm{~h}$ in an incubator at $37^{\circ} \mathrm{C}$. Antibacterial activity was defined as the diameter $(\mathrm{mm})$ of the clear inhibitory zone formed around the discs. MBC was defined as the lowest concentration that induced the clear inhibitory zone formed around the discs.

\section{RESULTS}

Scanning electron microscopic (SEM) studies of chitosan nanoparticles

The SEM micrograph details of the pure chitosan and chitosan nanoparticles was represented in Figures 1. The SEM micrograph obtained for 

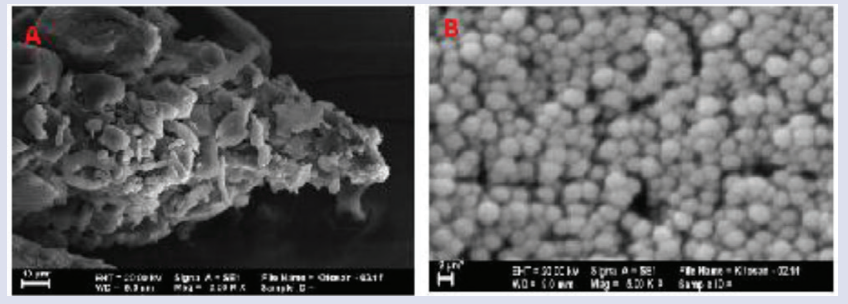

Figure 1: Scanning electron microscopic (SEM) of chitosan (A) and chitosan nanoparticles (B).

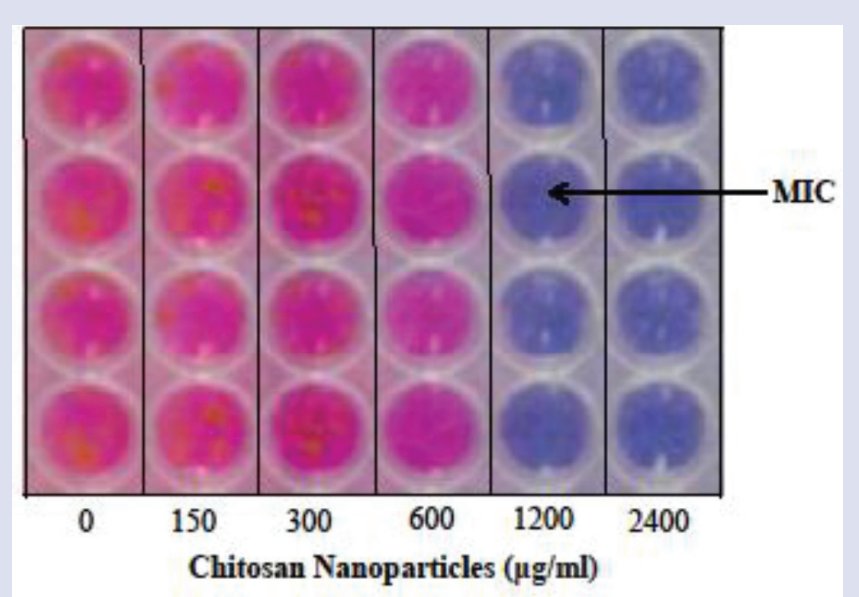

Figure 2: MIC of chitosan nanoparticles against Mycobacterium tuberculosis H37Rv on Resazurin Microtiter Plate Assay (REMA) method was $1200 \mu \mathrm{g} / \mathrm{mL}$. Serial four-fold dilutions of chitosan nanoparticles at dose $0 \mu \mathrm{g} / \mathrm{mL} ; 150 \mu \mathrm{g} / \mathrm{mL} ; 300 \mu \mathrm{g} / \mathrm{mL} ; 600 \mu \mathrm{g} / \mathrm{mL} ; 1200 \mu \mathrm{g} / \mathrm{ml}$; and $2400 \mu \mathrm{g} /$ $\mathrm{mL}$.

the pure chitosan revealed that the texture is plain without pores having smooth, compact and homogeneous even surface structure with no gross effects, while the SEM micrograph of chitosan nanoparticles Figure 1 revealed the rough surface morphology, have a solid dense cubical or rectangular structure and not aggregated. The spheres have mean diameters around $500 \mathrm{~nm}$. The nanoparticles dry powder consists of individual nanoparticles, which touch each other, but retain their original size and shape. The size variation may be related to different conditions of sample preparation for SEM.

\section{The MIC of the chitosan nanoparticles against} Mycobacterium tuberculosis on Resazurin Microtiter Plate Assay (REMA) method

The MIC of the chitosan nanoparticles was determined for their antimycobacterial activity using resazurin as an indicator of $M$. tuberculosis viability in 96-well microplates. The investigation showed that chitosan nanoparticles was active against $M$. tuberculosis. In this study, MIC of chitosan nanoparticles against Mycobacterium tuberculosis strains $\mathrm{H} 37 \mathrm{Rv}$ was $1200 \mu \mathrm{g} / \mathrm{mL}$ Figure 2.

\section{The MBC of the chitosan nanoparticles against}

\section{Mycobacterium tuberculosis on the paper disc method}

The antimycobacterial activity of chitosan nanoparticles against Mycobacterium tuberculosis strains $\mathrm{H} 37 \mathrm{Rv}$ was done using the paper disc method. MBC was defined as the lowest concentration that induced the clear inhibitory zone formed around the discs. The MBC of chitosan

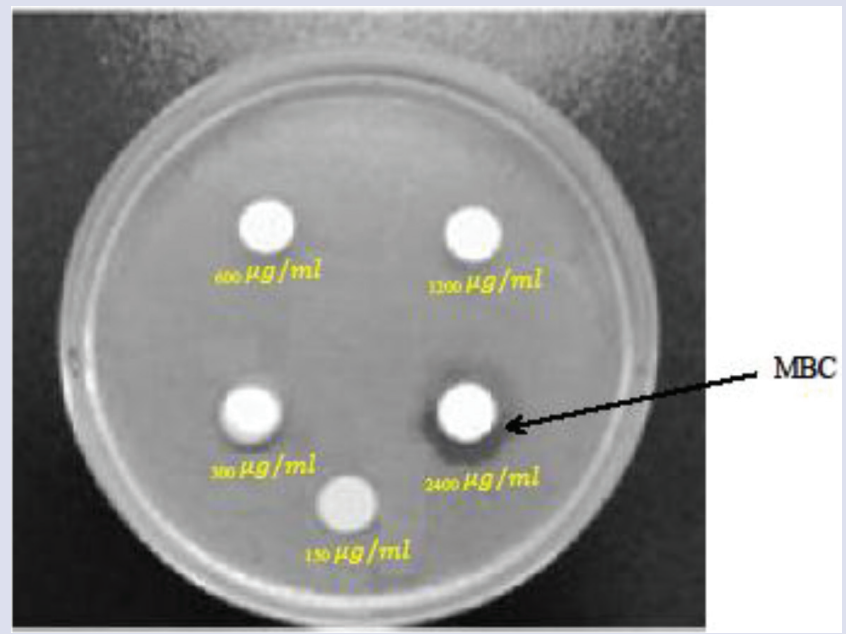

Figure 3: $M B C$ of chitosan nanoparticles against Mycobacterium tuberculosis H37Rv on the paper disc method.

nanoparticles against Mycobacterium tuberculosis strains $\mathrm{H} 37 \mathrm{Rv}$ was $2400 \mu \mathrm{g} / \mathrm{mL}$, which induced the clear inhibitory zone formed around the discs

\section{DISCUSSION}

Tuberculosis is a chronic disease caused by Mycobacterium tuberculosis. The emergence of antibiotic-resistant strains of this species underscores the need for novel effective drugs against resistant mycobacteria as first-line antituberculosis medications. ${ }^{1}$ The uses of natural product as traditional medicines are accepted, particularly in developing countries. ${ }^{2}$ This led us to the investigation of the effects of chitosan nanoparticles from shrimp on antimycobacterial activity.

We modified chitosan into chitosan nanoparticles with used sodium tripolyphosphate to prepare chitosan nanoparticles by ionotropic gelation method, which have more advantages over chitosan. Due to this modification can improve biodistribution and increase specificity and sensitivity, and reduced pharmacological toxicity. ${ }^{17}$ The topography, surfaces, structures, morphologies, and composition of the chitosan nanoparticles were studied using Scanning Electron Microscopic (SEM). ${ }^{18}$ The SEM micrograph obtained for the pure chitosan revealed that the texture is plain without pores having smooth, compact and homogeneous even surface structure with no gross effects. While the SEM micrograph of chitosan nanoparticles revealed the rough surface morphology. The SEM picture of chitosan nanoparticles demonstrates the good dispersion of the nanoparticles which are entangled one on the other with a larger exposed surface making the material very suitable for adsorption.

In the present study, we successfully synthesized and optimized chitosan nanoparticle. On comparing the SEM micrograph details of chitosan nanoparticles with pure chitosan, it was observed that the chitosan nanoparticles have a relatively rougher surface with uneven structure which exhibited highly amorphous feature. Several authors reported that the chitosan nanoparticles prepared from chitosan were found to be having the spherical shape. ${ }^{15}$

Particle size has an important role in obtaining optimal In vitro efficacy of chitosan nanoparticles. Particle size also had a crucial impact on the in vivo fate of a drug delivery system. Decreasing particle size could increase the dissolution and thus increase the bioavailability of poorly water soluble molecules. ${ }^{18,23}$ In our study, the chitosan nanoparticles have mean diameters around $500 \mathrm{~nm}$. The smaller size particles have efficient interfacial interaction with the cell membrane compared to larger size 
particles due to the endocytosis of small size particles. Small size particles could improve the efficacy of the particle-based oral drug delivery systems. Also, the use of small particle size can increase the bioavailability and prolong the blood half-life and increase the efficacy of drugs has been obtained.

Investigation of MIC and MBC plays an important role during the process of screening, prioritizing, and optimizing a chemical series during early drug discovery. Minimum Inhibitory Concentration determination by using Resazurin Microtiter Plate Assay (REMA) method. Many researchers have used the Resazurin Microtiter Plate Assay (REMA) method to screen test substances for antimycobacterial activity against M. tuberculosis. ${ }^{19}$ Resazurin, an oxidation-reduction indicator, has been used to assess viability and bacterial contamination and to test for antimicrobial activity. Results obtained using the REMA assay is faster and less expensive. Bearing in mind considerations of rapidity, low technology requirements and low cost, microplate assays that use Resazurin type compounds have the potential of becoming the methods of choice for drug susceptibility testing of $M$. tuberculosis in places where TB is a major problem. ${ }^{20}$ The $\mathrm{MBC}$ of the chitosan nanoparticles against Mycobacterium tuberculosis was conducted on the paper disc method. In this study, MIC of chitosan nanoparticles against Mycobacterium tuberculosis strains $\mathrm{H} 37 \mathrm{Rv}$ was $1200 \mu \mathrm{g} / \mathrm{mL}$, while MBC of chitosan nanoparticles against Mycobacterium tuberculosis strains $\mathrm{H} 37 \mathrm{Rv}$ was $2400 \mu \mathrm{g} / \mathrm{mL}$. These results are in agreement with reports in the literature that have documented the antibacterial activity of chitosan against a large number of g-positive and g-negative bacteria. Some researcher has also shown that chitosan generally showed stronger effects for g-positive bacteria (e.g. Listeria monocytogenes, Bacillus megaterium, B. cereus, Staphylococcus aureus, Lactobacillus plantarum, L. brevis, L. bulgaris, etc.) and for g-negative bacteria (e.g. E. coli, Pseudomonas fluorescens, Salmonella typhymurium, Vibrio parahaemolyticus, etc.). ${ }^{7,8,14}$ The polycationic characteristic of chitosan in acidic medium is the main factor involving to the antimicrobial activity. Due to the positive surface charges at acidic condition, chitosan interacts with anionic components on bacteria surface, such as peptidoglycan and teichoic acid in cell wall of G-positive bacteria, and negatively charged lipopolysaccharide in outer membrane of G-negative bacteria. The chitosan molecules be able to pass through the bacterial cell wall, composed of multilayers of cross-linked murein, and reach the plasma membrane. This effect due to the electrostatic interactions between chitosan molecules and microbial cell membranes, leading to the leakage of proteinaceous and consequently increased chitosan penetration to nucleus and binding to microbial DNA, which can inhibit mRNA and protein synthesis. ${ }^{710,14,24}$ This suggests that chitosan has potent activity as antibacterial and this has been confirmed experimentally.

\section{CONCLUSION}

The findings from this research can be concluded that chitosan nanoparticles have a relatively rougher surface with an uneven structure which exhibited highly amorphous feature, and it has promising anti-tubercular activity by preliminary In vitro techniques. Therefore, it has the definite potential as a source of compounds that may be developed further into antimycobacterial drugs.

\section{ACKNOWLEDGEMENT}

This study was supported by Ministry of Research, Technology and Higher Education of the Republic of Indonesia. Grants No: 120/Sp2H/ LT/DRPM/IV/2017

\section{CONFLICT OF INTEREST}

Authors declare no conflict of interest.

\section{ABBREVIATIONS USED}

TPP: Tripolyphosphate; SEM: Scanning Electron Microscope; MIC: Minimal Inhibitory Concentration; MBC: Minimal Bactericidal Concentration; TB: Tuberculosis; MDR-TB: Multi Drug Resistant TB; XDRTB: Extensively Drug Resistant TB); REMA: Resazurin Microtiter Plate Assay.

\section{REFERENCES}

1. World Health Organization. Global Tuberculosis Report 2015. 20 Avenue Appia, 1211 Geneva 27, Switzerland: World Health Organization; 2015. p. 1-145.

2. Cragg GM, Newman DJ. Natural Products: A Continuing Source of Novel Drug Leads. Biochim Biophys Acta. 2013;1830(6):3670-95. doi: 10.1016/j.bbagen.2013.02.008. Epub 2013 Feb 18.

3. Njeru SN, Obonyo MA, Ngari SM, Njeru JM, Kamweru PP. Antituberculous, antimicrobial, cytotoxicity and phytochemical activity study of Piliostigma thonningii extract fractions. J. Med Plants Res. 2015;9(22):655-63. http://dx.doi. org/10.5897/JMPR2015.5822.

4. Sudjarwo SA, Eraiko K, Wardani G, Koerniasari. The potency of chicken egg yolk immunoglobulin (lgY) specific as immunotherapy to Mycobacterium tuberculosis infection. J Adv Pharm Technol Res. 2017;8(3):91-6. 10.4103/japtr. JAPTR-167-16.

5. Fadipe VO, Opoku AR, Dikhoba PM, Makhafola TJ. Isolation of anti-mycobacterial compounds from Curtisia dentata (Burm.f.) C.A.Sm (Curtisiaceae); Bio Med Central Complement Altern Med. 2017;17(1):306-13. http://dx.doi.org/10.1186/ s12906-017-1818-9.

6. Younes I, Rinaudo M. Chitin and chitosan preparation from marine sources. Structure, properties and applications. Marine Drugs. 2015;13(3):1133-74. http:// dx.doi.org/10.3390/md13031133.

7. Vilar JC, Ribeaux DR, Silva CAA, Takaki GM. Physicochemical and Antibacterial Properties of Chitosan Extracted from Waste Shrimp Shells. Inter J Microbiol. 2016;1-7. http://dx.doi.org/10.1155/2016/5127515.

8. Kong M, Chen XG, Xing K, Park HY. Antimicrobial properties of chitosan and mode of action: A state of the art review. Int J Food Microbiol. 2010;144(1):5163. http://dx.doi.org/10.1016/j.ijfoodmicro.2010.09.012.

9. Goya RC, Britto D, Assis OBG. A review of the antimicrobial activity of chitosan. Polímeros. 2009;19(3):241-7. http://dx.doi.org/10.1590/S010414282009000300013.

10. Ngamviriyavong $P$, Thananuson $A$, Pankongadisak $P$, Tanjak $P$, Janvikul $W$. Antibacterial hydrogels from chitosan derivatives. J Met Mater Miner. 2010; 20(3):113-7.

11. Kaya M, Baran T, Asan-Ozusaglam M. Extraction and characterization of chitin and chitosan with antimicrobial and antioxidant activities from cosmopolitan Orthoptera species. Biotechnol Bioprocess Eng. 2015;20(1):168-79. http:// dx.doi.org/10.1007/s12257-014-0391.

12. Friedman M, Juneja VK. Review of antimicrobial and antioxidative activities of chitosans in food. J Food Prot. 2010;73(9):1737-61. http://dx.doi.org/10.4315/ 0362-028X-73.9.1737.

13. Ahmed TA, Aljaeid BM. Preparation, characterization, and potential application of chitosan, chitosan derivatives, and chitosan metal nanoparticles in pharmaceutical drug delivery. Drug Des Dev Ther. 2016;10:483-507. http://dx.doi org/10.2147/DDDT.S99651. PMCID: PMC4734734.

14. Qi L, Xu Z, Jiang X, Hu C, Zou X. Preparation and antibacterial activity of chitosan nanoparticles," Carbohydrate Res. 2004;339(16):2693-700. http:// dx.doi.org/10.1016/j.carres.2004.09.007.

15. Ghadi A, Mahjoub S, Tabandeh F, Talebnia F. Synthesis and optimization of Chitosan nanoparticles: Potential applications in nanomedicine and biomedical engineering Iran. Caspian J Intern Med. 2014;5(3):156-61. PMID:25202443 PMCID: PMC4143737.

16. Saini R, Saini S, Sharma S. Nanotechnology: The Future Medicine. J Cutan Aesthet Surg. 2010;3(1):32-3. http://dx.doi.org/10.4103/0974-2077.63301.

17. Vimal S, Majeed SA, Taju G, Farook MA, RajkumarT, Gopinath D, et al. Chitosan tripolyphosphate (CS/TPP) nanoparticles: Preparation, characterization and application for gene delivery in shrimp. Acta Tropica. 2013;128(3):486-93. http:// dx.doi.org/10.1016/j.actatropica.2013.07.013.

18. Khanmohammadi M, Elmizadeh H, Ghasemi K. Investigation of Size and Morphology of Chitosan Nanoparticles Used in Drug Delivery System Employing Chemometric Technique. Iranian J Pharmaceutic Res. 2015;14(3):665-75.

19. Palomino JC, Martin A, Camacho M, Guerra H, Swings J, Portaels F. Resazurin microtiter assay plate: simple and inexpensive method for detection of drug resistance. Antimicrob Agents Chemother. 2002;46(8):2720-2. http://dx.doi. org/10.1128/AAC.46.8.2720-2722.

20. Ang CF, Mendoza MT, Bulatao WC. Evaluation of the Resazurin Microtiter Assay for Drug Susceptibility Testing of Clinical Isolates of Mycobacterium tuberculosis. Philipp J Microbiol Infect Dis. 2010;39:59-65. http://dx.doi.org/10.1016/j. mimet.2011.11.006. 
21. Nyambuya T, Mautsa R, Mukanganyama S, Alkaloid extracts from Combretum zeyheri inhibit the growth of Mycobacterium smegmatis. Bio Med Central Complement Altern Med. 2017;17(1):124-30. http://dx.doi.org/10.1186/s12906017-1636-0.

22. Primm TP, Franzblau SG. Recent Advances in Methodologies for the Discovery of Antimycobacterial Drugs. Curr Bioactive Compounds. 2007;3(3):201-8. http:// dx.doi.org/10.2174/157340707781695550.
23. Sudheesh K, Mishra AK, Arotiba OA, Mamba BB. Chitosan-based nanomaterials: A state-of-the-art review. Int J Biol Macromolec. 2013;59:46-58. http://dx.doi org/10.1016/j.ijbiomac.2013.04.043.

24. Chung YC, SuYP, Chen CC. Relationship between antibacterial activity of chitosan and surface characteristics of cell wall," Acta Pharmacol Sin. 2004;25(7):932-6. PMID:15210068.

\section{GRAPHICAL ABSTRACT}

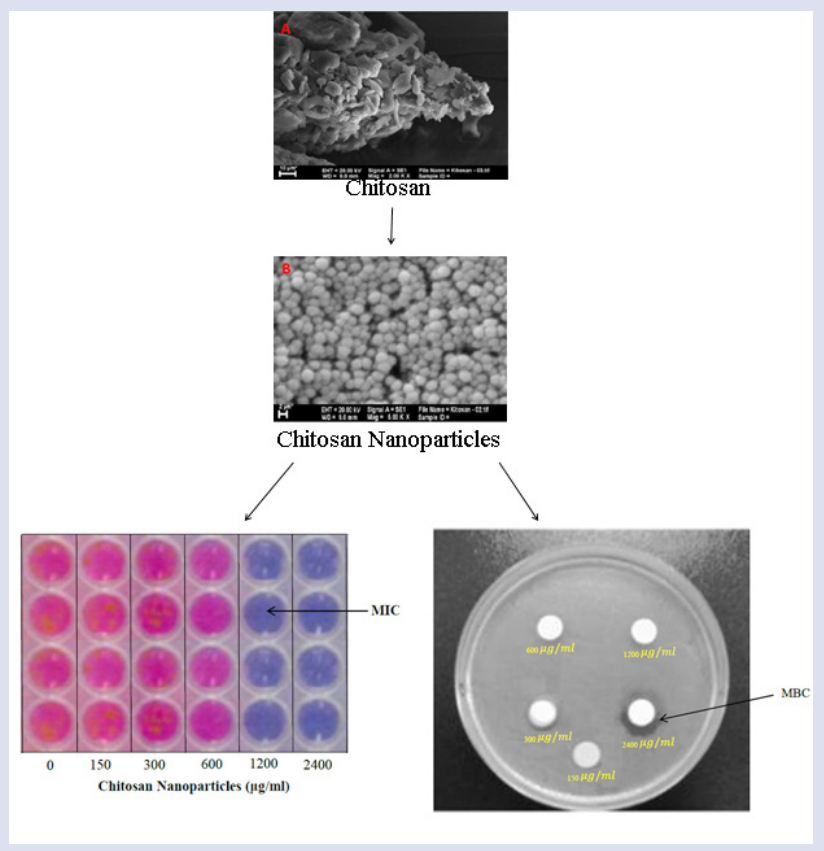

\section{SUMMARY}

- The chitosan nanoparticles were synthesized from the chitosan using sodium tripolyphosphate by ionotropic gelation method

- The SEM micrographs of chitosan nanoparticles showed that they were approximately uniform spheres and the rough surface morphology, have a solid dense cubical or rectangular structure.

- The chitosan nanoparticle have the antimycobacterial effects with a MIC value of $1200 \mu \mathrm{g} / \mathrm{mL}$ and MBCs value of $2400 \mu \mathrm{g} / \mathrm{mL}$ for M. tuberculosis H37Rv

- The chitosan nanoparticle have potential as a source of compounds that may be developed further into antimycobacterial drugs

\section{ABOUT AUTHORS}

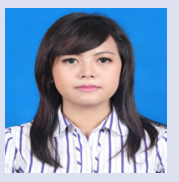

Giftania Wardani: Lecturer at the Department of Pharmacy Biology, Faculty of Pharmacy, Hang Tuah University, Surabaya, Indonesia. where she graduated in Magister Degree at the Department of Molecular Biotechnology, Faculty of Medicine, Konkuk University, Republic of Shouth Korea. Her research focused on Pharmacology and Toxicology.

Cite this article: Wardani G, Mahmiah, Sudjarwo SA. In vitro Antibacterial Activity of Chitosan Nanoparticles against Mycobacterium tuberculosis. Pharmacog J. 2018;10(1):162-6. 